\begin{tabular}{l|c|c}
\hline ISSN: 0001-5113 & ACTA ADRIAT., & ORIGINAL SCIENTIFIC PAPER \\
AADRAY & $62(1): 63-73,2021$ & \\
\hline \hline
\end{tabular}

\title{
Checklist of marine fishes of Albania
}

\author{
Alen $\mathrm{SOLDO}^{1}$ and Rigers BAKIU ${ }^{2,3}$ \\ ${ }^{1}$ Department of Marine Studies, University of Split, Croatia \\ ${ }^{2}$ Department of Aquaculture and Fisheries, Faculty of Agriculture and Environment, \\ Agricultural University of Tirana, Koder-Kamez, Albania \\ ${ }^{3}$ ACEPSD, Albanian Center for Environmental Protection and Sustainable Development, \\ Tirana, Albania
}

*Corresponding author, email: soldo@unist.hr

This paper presents an updated checklist of marine fishes of Albania and the first one published in the English language. The checklist contains 262 species compiled from published literature and personal surveys.

Key words: checklist; fish; Albania; Adriatic; Ionian Sea

\section{INTRODUCTION}

The Albanian coastline has a total length of about $380 \mathrm{~km}$ (excluding the internal shore of coastal lagoons), with about $284 \mathrm{~km}$ stretching along the South Adriatic Sea and the remaining $96 \mathrm{~km}$, south of the Gulf of Vlore, facing the North Ionian Sea. The two seas have quite distinct physical and chemical characteristics: the Adriatic Sea shows large seasonal variations in temperature and productivity, with levels of nutrients and salinity largely controlled by freshwater inputs. The Ionian Sea has instead a more uniform physical and chemical oceanography throughout the year (SIMEONI et al., 1997).

The Albanian littoral is of a particular interest from a bio-geographical point of view, as it is located near or on the boundaries of three bio-geographical subdivisions: Eastern Mediterranean, Western Mediterranean and the Adriatic (GJIKNURI, 1995). However, fish fauna was very rarely investigated and the first and only list of marine fishes recorded in Albania was made in 1995 when a total of 250 species was counted (RAKAJ, 1995). Hence, as the list was published in the Albanian language it was rarely used and acknowledged by the international scientific community. Considering the constant and rapid change of climatic and other environmental factors that are influencing ichthyofauna assemblages, it is not surprising that a rising number of new records of fish was observed in all Mediterranean, e.g. in the Adriatic the number of species from 1996 to 2010 was raised by 33 new fish species and 23 new species were added to the list from 2010 to 2020 (LIPEJ \& DULČIĆ, 2010; KOVAČIĆ et al., 2020). Therefore, the main objective of this paper is to present the updated checklist of marine fishes of Albania after the period of 25 years since the last one (RAKAJ, 1995), which will be, also, the first one published in the English language and the first one broadly available considering limited edition in Albanian language of RAKAJ's (1995) book. 


\section{MATERIAL AND METHODS}

Orders and families were arranged according to Eschmeyer's Catalog of Fishes (FRICKE et al., 2020; VAN DER LAAN et al., 2020) and Fishbase (www.fishbase.com). Only those marine species which were documented in the scientific literature were taken into consideration. Hence, fish species with typical freshwater habits were excluded from the list while species from brackish waters were included as they were originally reported in RAKAJ's (1995) list.

This checklist of marine fishes of Albania is compiled using a previous list of RAKAJ (1995) and new records and evidence of fish species by checking ichthyological scientific literature and by recent ichthyofauna surveys conducted by authors of this paper.

\section{RESULTS AND DISCUSSION}

The total number of 262 fish species inhabiting the seas of Albania was counted (Agnatha 1, Elasmobranchii 51, Holocephali 1 and Actinopterygii 209) from 106 fish families increasing the known fish diversity for 12 species compared to the last review in RAKAJ (1995) where 250 species were reported. Six of these species have published records for Albanian waters in the last few years (i.e. 2017-2019), while six of them are the first records in Albania originally reported in the present work. Out of this number 19 species from this list are not listed in the recently revised checklist of the Adriatic species (KOVAČIĆ et al., 2020) which can be explained by the bordering position of the southern part of Albania between two seas, the Adriatic and the Ionian. E.g. Carcharhinus melanopterus (Quoy \& Gaimard, 1824) and Diplodus cervinus (Lowe, 1838) are typically species that are inhabiting warmer waters than it is the Adriatic, while three cyprinid species (Cyprinidae) are recorded in the southern Albanian brackish lagoons. Even higher number of species that are not recorded in the Adriatic can be counted if we compare this list with the checklist of LIPEJ \& DULČIĆ (2010). This fact can be used as support to a common opinion that Albania shares the
Adriatic and the Ionian sea. However, KOVAČIĆ et al. (2020) used some records of the species that have been recorded only in the southern Albanian areas (e.g. Hexanchus nakamurai Teng, 1962) which are usually considered as the part of the Ionian Sea, and listed them as the Adriatic species due to the certain opinions that the border between the Adriatic and Ionian Sea is more south which results with a situation that whole Albanian waters are part of the Adriatic only and not the Ionian Sea (IHO, 1953). Nevertheless, it is obvious that this new checklist of the Albanian marine fishes will impact the Adriatic checklist and probably the Ionian. Also, it has to be noted that the number of the Albanian fishes is considerably lower than the number of Adriatic species as LIPEJ \& DULČIĆ (2010) listed 440 species while KOVAČIĆ et al. (2020) ten years after confirmed, using evidence approach, 444 species out of 466 reported species. Although such a high difference may be explained by different geographical and geomorphological factors it is more likely a result of a lack of focused fish surveys in Albania. E.g. KOVAČIĆ et al. (2020) listed 46 goby species and 16 blenny species for the Adriatic while this list reports only 3 goby and 6 blenny species. Moreover, a large portion of deepwater species is missing in the Albanian list. Thus, it can be concluded that the current list can be easily updated with a new species from the future surveys focusing more on some particular habitats which are not traditional fishing grounds, from where most of this list is compiled, such as deep waters and very shallow sandy and rocky bottoms. Hence, a critical assessment of each species' presence following a protocol of the evidence approach proposed by KOVAČIĆ et al. (2020) would also be beneficial. 
List of marine fish species of Albania

CLASS: PETROMYZONTI

ORDER: PETROMYZONTIFORMES PETROMYZONTIDAE

PETROMYZON Linnaeus, 1758

1. Petromyzon marinus Linnaeus, 1758

\section{CLASS: ELASMOBRANCHII}

ORDER: HEXANCHIFORMES

HEXANCHIDAE

HEPTRANCHIAS Rafinesque \& Schmaltz, 1810

2. Heptranchias perlo (Bonnaterre, 1788)

HEXANCHUS Rafinesque \& Schmaltz, 1810

3. Hexanchus griseus (Bonnaterre, 1788)

4. Hexanchus nakamurai Teng, $1962 * 1$

\section{ORDER: LAMNIFORMES}

\section{CARCHARIIDAE}

CARCHARIAS Rafinesque, 1810

5. Carcharias taurus Rafinesque, 1810

\section{ODONTASPIDIDAE}

ODONTASPIS Agassiz, 1838

6. Odontaspis ferox (Risso, 1810)

\section{LAMNIDAE}

CARCHARODON Smith, 1838

7. Carcharodon carcharias (Linnaeus, 1758)

ISURUS Rafinesque, 1810

8. Isurus oxyrinchus Rafinesque, 1810

LAMNA Cuvier, 1817

9. Lamna nasus (Bonnaterre, 1788)

\section{CETORHINIDAE}

CETORHINUS Blainville, 1816

10. Cetorhinus maximus (Günnerus, 1765)

\section{ALOPIIDAE}

ALOPIAS Rafinesque, 1810

11. Alopias vulpinus (Bonnaterre, 1788)

12. Alopias superciliosus Lowe, $1841^{* 2}$

ORDER: CARCHARHINIFORMES

PENTANCHIDAE

GALEUS Rafinesque, 1810

13. Galeus melastomus Rafinesque, 1810

\section{SCYLIORHINIDAE}

SCYLIORHINUS Blainville, 1816

14. Scyliorhinus canicula (Linnaeus, 1758)

15. Scyliorhinus stellaris (Linnaeus, 1758)

TRIAKIDAE

GALEORHINUS Blainville, 1816

16. Galeorhinus galeus (Linnaeus, 1758)

MUSTELUS Linck, 1790

17. Mustelus asterias Cloquet, 1821

18. Mustelus mustelus (Linnaeus, 1758)

19. Mustelus punctulatus Risso, $1827^{*}$

\section{CARCHARHINIDAE}

CARCHARHINUS Blainville, 1816

20. Carcharhinus melanopterus (Quoy \& Gaimard, 1824)

21. Carcharhinus plumbeus (Nardo, 1827)

RHIZOPRIONODON Whitley, 1929

22. Rhizoprionodon acutus (Rüppell, 1837)*3

PRIONACE Cantor, 1849

23. Prionace glauca (Linnaeus, 1758)

\section{SPHYRNIDAE}

SPHYRNA Rafinesque, 1810

24. Sphyrna zygaena (Linnaeus, 1758)

ORDER: SQUALIFORMES

DALATIIDAE

DALATIAS Rafinesque, 1810

25. Dalatias licha (Bonnaterre, 1778)

ETMOPTERIDAE

ETMOPTERUS Rafinesque, 1810

26. Etmopterus spinax (Linnaeus, 1758)

\section{OXYNOTIDAE}

OXYNOTUS Rafinesque, 1810

27. Oxynotus centrina (Linnaeus, 1758)

\section{CENTROPHORIDAE}

CENTROPHORUS Müller \& Henle, 1841

28. Centrophorus granulosus (Bloch \& Schneider, 1801)

SQUALIDAE

SQUALUS Linnaeus, 1758

29. Squalus acanthias Linnaeus, 1758

30. Squalus blainvillei (Risso, 1827) 


\section{ORDER: ECHINORHINIFORMES}

\section{ECHINORHINIDAE}

ECHINORHINUS Blainville, 1816

31. Echinorhinus brucus (Bonnaterre, 1788)

\section{ORDER: SQUATINIFORMES}

SQUATINIDAE

SQUATINA Duméril, 1810

32. Squatina aculeata Cuvier, 1829

33. Squatina squatina (Linnaeus, 1758)

\section{ORDER: TORPEDINIFORMES}

\section{RHINOBATIDAE}

RHINOBATOS Klein, 1776

34. Rhinobatos rhinobatos (Linnaeus, 1758)

\section{TORPEDINIDAE}

TORPEDO Houttuyn, 1764

35. Torpedo marmorata Risso, 1810

36. Torpedo torpedo (Linnaeus, 1758)

TETRONARCE Gill, 1862

37. Tetronarce nobiliana (Bonaparte, 1835) *4

\section{ORDER: RAJIFORMES}

RAJIDAE

DIPTURUS Rafinesque, 1810

38. Dipturus batis (Linnaeus, 1758)

39. Dipturus oxyrinchus (Linnaeus, 1758)

LEUCORAJA Malm, 1877

40. Leucoraja fullonica (Linnaeus, 1758)

RAJA Linnaeus, 1758

41. Raja asterias Delaroche, 1809

42. Raja clavata Linnaeus, 1758

43. Raja miraletus Linnaeus, 1758

44. Raja radula Delaroche, 1809

45. Raja undulata Lacepède, 1803

ROSTRORAJA Hulley, 1758

46. Rostroraja alba Lacepède, 1803

ORDER: MYLIOBATIFORMES

\section{DASYATIDAE}

BATHYTOSHIA Whitley, 1933

47. Bathytoshia centroura (Mitchill, 1815)

DASYATIS Rafinesque, 1810

48. Dasyatis pastinaca (Linnaeus, 1758)

\section{GYMNURIDAE}

GYMNURA Van Hasselt, 1823

49. Gymnura altavela (Linnaeus, 1758)

\section{MYLIOBATIDAE}

MYLIOBATIS Cuvier, 1816

50. Myliobatis aquila (Linnaeus, 1758)

AETOMYLAEUS Garman, 1908

51. Aetomylaeus bovinus (Geoffroy St.Hilaire, 1817)*

\section{MOBULIDAE}

MOBULA Rafinesque, 1810

52. Mobula mobular (Bonnaterre, 1788)

\section{CLASS: HOLOCEPHALI}

ORDER: CHIMAERIFORMES

\section{CHIMAERIDAE}

CHIMAERA Linnaeus, 1758

53. Chimaera monstrosa Linnaeus, 1758

CLASS: ACTINOPTERYGII = OSTEICHTHYES

\section{ORDER: ACIPENSERIFORMES}

\section{ACIPENSERIDAE}

ACIPENSER [Artedi, 1738] Linnaeus, 1758

54. Acipenser naccarii Bonaparte, 1830

55. Acipenser stellatus Pallas, 1771

56. Acipenser sturio Linnaeus, 1758

HUSO Brandt, 1869

57. Huso huso (Linnaeus, 1758)

\section{ORDER: ANGUILLIFORMES}

ANGUILLIDAE

ANGUILLA Schrank, 1798

58. Anguilla anguilla (Linnaeus, 1758)

\section{CHLOPSIDAE}

CHLOPSIS Rafinesque, 1810

59. Chlopsis bicolor Rafinesque, 1810

MURAENIDAE

MURAENA Linnaeus, 1758

60. Muraena helena Linnaeus, 1758

\section{OPHICHTIDAE}

DALOPHIS Rafinesque, 1810

61. Dalophis imberbis (Delaroche, 1809)

ECHELUS Rafinesque, 1810

62. Echelus myrus (Linnaeus, 1758) 
OPHISURUS Lacepède, 1800

63. Ophisurus serpens Linnaeus, 1758

CONGRIDAE

CONGER Oken, 1817

64. Conger conger (Linnaeus, 1758)

ORDER: CLUPEIFORMES

CLUPEIDAE

ALOSA Linck, 1790

65. Alosa agone (Scopoli, 1786)

66. Alosa fallax (Lacepède, 1803)

SARDINA Antipa, 1906

67. Sardina pilchardus (Walbaum, 1792)

SARDINELLA Valenciennes, 1847

68. Sardinella aurita Valenciennes, 1847

SPRATTUS Girgensohn, 1846

69. Sprattus sprattus (Linnaeus, 1758)

ENGRAULIDAE

ENGRAULIS Cuvier, 1816

70. Engraulis encrasicolus (Linnaeus, 1758)

ORDER: CYPRINIFORMES

CYPRINIDAE

ALBURNUS Rafinesque, 1820

71. Alburnus chalcoides (Güldenstädt, 1772)

SCARDINIUS (subgenus of Leuciscus) Bonaparte, 1837

72. Scardinius scardafa (Bonaparte, 1837)

TINCA Garsault, 1764

73. Tinca tinca (Linnaeus, 1758)

ORDER: ARGENTINIFORMES

ARGENTINIDAE

ARGENTINA Linnaeus, 1758

74. Argentina sphyraena Linnaeus, 1758

GLOSSANODON Guichenot, 1867

75. Glossanodon leioglossus (Valenciennes, 1848)

\section{MICROSTOMATIDAE}

Microstoma Cuvier, 1816

76. Microstoma microstoma (Risso, 1810)

NANSENIA Jordan \& Evermann, 1896

77. Nansenia oblita (Facciola, 1887)
ORDER: AULOPIFORMES

\section{AULOPIDAE}

AULOPUS Cloquet, 1816

78. Aulopus filamentosus (Bloch, 1792)

\section{CHLOROPHTALMIDAE}

CHLOROPTHALMUS Bonaparte, 1840

79. Chlorophthalmus agassizii Bonaparte, 1840

SYNODONTIDAE

SAURIDA Valenciennes, 1849

80. Saurida lessepsianus Russell, Golani \& Tikochinski, 2015

SYNODUS Scopoli, 1777

81. Synodus saurus (Linnaeus, 1758)

\section{ORDER: LAMPRIFORMES}

\section{TRACHIPTERIDAE}

TRACHIPTERUS Goian, 1770

82. Trachipterus trachypterus (Gmelin, 1789)

ZU Walters \& Fitch, 1960

83. Zu cristatus (Bonelli, 1829)

\section{ORDER: TRACHICHTHYFORMES}

TRACHICHTHYIDAE

HOPLOSTETHUS Cuvier, 1829

84. Hoplostethus mediterraneus Cuvier, 1829*

ORDER: GADIFORMES

\section{GADIDAE}

MICROMESISTIUS Gill, 1863

85. Micromesistius poutassou (Risso, 1826)

TRISOPTERUS Rafinesque, 1814

86. Trisopterus capelanus (Lacepède, 1800)

\section{LOTIDAE}

GAIDROPSARUS Rafinesque, 1810

87. Gaidropsarus mediterraneus (Linnaeus, 1758)

MACROURIDAE

COELORINCHUS Giorna, 1809

88. Coelorinchus caelorhincus (Risso, 1810)

MERLUCCIIDAE

MERLUCCIUS Rafinesque, 1810

89. Merluccius merluccius (Linnaeus, 1758)

PHYCIDAE 
PHYCIS Artedi, 1792

90. Phycis blennoides (Brünnich, 1768)

91. Phycis phycis (Linnaeus, 1758)

ORDER: OPHIDIIFORMES

OPHIDIIDAE

OPHIDION Linnaeus, 1758

92. Ophidion barbatum Linnaeus, 1758

93. Ophidion rochei Müller, 1845

PAROPHIDION Tortonese, 1954

94. Parophidion vassali (Risso, 1810)

\section{CARAPIDAE}

CARAPUS Rafinesque, 1810

95. Carapus acus (Brünnich, 1768)

ORDER LOPHIIFORMES

LOPHIIDAE

LOPHIUS Linnaeus, 1758

96. Lophius budegassa Spinola, 1807

97. Lophius piscatorius Linnaeus, 1758

ORDER: GOBIESOCIFORMES

GOBIESOCIDAE

LEPADOGASTER Goüan, 1770

98. Lepadogaster lepadogaster (Bonnaterre, 1788)

ORDER: ATHERINIFORMES

ATHERINIDAE

ATHERINA Linnaeus, 1758

99. Atherina boyeri Risso, 1810

100. Atherina hepsetus Linnaeus, 1758

ORDER: CYPRINODONTIFORMES

CYPRINODONTIDAE

APHANIUS Nardo, 1827

101. Aphanius fasciatus Nardo, 1827

102. Aphanius dispar (Rüppell, 1829)

103. Aphanius iberus (Valenciennes, 1846)

POECILIIDAE

POECILIA Bloch \& Schneider, 1801

104. Poecilia reticulata Peters, 1859

ORDER: BELONIIFORMES

\section{BELONIDAE}

BELONE Cuvier, 1817

105. Belone belone (Linnaeus, 1760)

\section{EXOCOETIDAE}

EXOCOETUS Linnaeus, 1758

106. Exocoetus volitans Linnaeus, 1758

CHEILOPOGON Lowe, 1840

107. Cheilopogon exsiliens (Linnaeus, 1771)

108. Cheilopogon heterurus (Rafinesque, 1810)

HIRUNDICHTHYS Breder, 1928

109. Hirundichthys rondeletii (Valenciennes, 1846)

\section{SCOMBERESOCIDAE}

SCOMBERESOX Lacepède, 1803

110. Scomberesox saurus (Walbaum, 1792)

\section{ORDER: MUGILIFORMES}

MUGILIDAE

CHELON Artedi, 1793

111. Chelon labrosus (Risso, 1827)

112. Chelon ramada (Risso, 1827)

LIZA Jordan \& Swain, 1884

113. Liza aurata (Risso, 1810)

114. Liza saliens (Risso, 1810)

MUGIL Linnaeus, 1758

115. Mugil cephalus Linnaeus, 1758

OEDALECHILUS Fowler,

116. Oedalechilus labeo (Cuvier, 1829)

ORDER: ZEIFORMES

ZEIDAE

ZEUS Linnaeus, 1758

117. Zeus faber Linnaeus, 1758

ORDER: ACANTHURIFORMES

CAPROIDAE

CAPROS Lacepède, 1802

118. Capros aper (Linnaeus, 1758)

ORDER: SYNGNATHIFORMES

CENTRISCIDAE

MACRORAMPHOSUS Lacepède, 1803

119. Macroramphosus scolopax (Linnaeus, 1758)

SYNGNATHIDAE

HIPPOCAMPUS Rafinesque, 1810

120. Hippocampus hippocampus (Linnaeus, 1758) 
121. Hippocampus guttulatus Cuvier, 1829

SYNGNATHUS Linnaeus, 1758

122. Syngnathus acus Linnaeus, 1758

123. Syngnathus tenuirostris Rathke, 1837

124. Syngnathus typhle Linnaeus, 1758

ORDER: SCORPAENIFORMES PERISTEDIIDAE

PERISTEDION Lacepède, 1801

125. Peristedion cataphractum (Linnaeus, 1758)

\section{SEBASTIDAE}

HELICOLENUS Goode \& Bean, 1896

126. Helicolenus dactylopterus (Delaroche, 1809)

\section{SCORPAENIDAE}

SCORPAENA Linnaeus, 1758

127. Scorpaena porcus Linnaeus, 1758

128. Scorpaena notata Rafinesque, 1810

129. Scorpaena scrofa Linnaeus, 1758

\section{DACTYLOPTERIDAE}

DACTYLOPTERUS Lacepède, 1801

130. Dactylopterus volitans (Linnaeus, 1758)

\section{TRIGLIDAE}

ASPITRIGLA Fowler, 1925

131. Aspitrigla cuculus (Linnaeus, 1758)

CHELIDONICHTHYS Kaup, 1873

132. Chelidonichthys lucerna (Linnaeus, 1758)

133. Chelidonichthys lastoviza (Bonnaterre, 1788)

134. Chelidonichthys obscurus (Bloch \& Schneider, 1801)

LEPIDOTRIGLA Günther, 1860

135. Lepidotrigla cavillone (Lacepède, 1801)

TRIGLA Linnaeus, 1758

136. Trigla lyra Linnaeus, 1758

\section{ORDER: PERCIFORMES}

\section{MORONIDAE}

DICENTRARCHUS Gill, 1860

137. Dicentrarchus labrax (Linnaeus, 1758)

138. Dicentrarchus punctatus (Bloch, 1792)

POLYPRIONIDAE

POLYPRION Oken, 1817

139. Polyprion americanus (Bloch \& Schneider, 1801)

\section{SERRANIDAE}

ANTHIAS Bloch, 1792

140. Anthias anthias (Linnaeus, 1758)

EPINEPHELUS Bloch, 1793

141. Epinephelus aeneus (Geoffroy Saint-Hilarie, 1817)

142. Epinephelus caninus (Valenciennes, 1843)

143. Epinephelus costae (E. Geoffroy, 1817)

144. Epinephelus marginatus (Lowe, 1834)

SERRANUS Cuvier, 1816

145. Serranus cabrilla (Linnaeus, 1758)

146. Serranus hepatus (Linnaeus, 1758)

147. Serranus scriba (Linnaeus, 1758)

\section{CALLANTHIDAE}

CALLANTHIAS Lowe, 1839

148. Callanthias ruber (Rafinesque, 1810)

APOGONIDAE

APOGON Lacepède, 1801

149. Apogon imberbis (Linnaeus, 1758)

\section{ECHENEIDAE}

REMORA Gill, 1862

150. Remora remora (Linnaeus, 1758)

151. Remora brachyptera (Lowe, 1839)

\section{CARANGIDAE}

LICHIA Cuvier, 1816

152. Lichia amia (Linnaeus, 1758)

NAUCRATES Rafinesque, 1810

153. Naucrates ductor (Linnaeus, 1758)

SERIOLA Cuvier, 1817

154. Seriola dumerili (Risso, 1810)

TRACHINOTUS Lacepède, 1801

155. Trachinotus ovatus (Linnaeus, 1758)

TRACHURUS Rafinesque, 1810

156. Trachurus mediterraneus (Steindachner, 1868)

157. Trachurus picturatus (T.E. Bowdich, 1825)

158. Trachurus trachurus (Linnaeus, 1758)

\section{CORYPHAENIDAE}

CORYPHAENA Linnaues, 1758

159. Coryphaena hippurus Linnaeus, 1758

\section{SPARIDAE}

BOOPS Cuvier, 1814 
160. Boops boops (Linnaeus, 1758)

DENTEX Cuvier, 1814

161. Dentex gibbosus (Rafionesque, 1810)

162. Dentex dentex (Linnaeus, 1758)

163. Dentex macrophthalmus (Bloch, 1791)

DIPLODUS Rafinesque, 1810

164. Diplodus annularis (Linnaeus, 1758)

165. Diplodus puntazzo (Cetti, 1777)

166. Diplodus sargus (Linnaeus, 1758)

167. Diplodus vulgaris (Geoffroy Saint-Hilaire, 1817)

168. Diplodus cervinus (Lowe, 1838)

LITHOGNATHUS Swainson, 1839

169. Lithognathus mormyrus (Linnaeus, 1758)

OBLADA Cuvier, 1829

170. Oblada melanura (Linnaeus, 1758)

PAGELLUS Valenciennes, 1830

171. Pagellus acarne (Risso, 1826)

172. Pagellus bogaraveo (Brünnich, 1768)

173. Pagellus erythrinus (Linnaues, 1758)

PAGRUS Cuvier, 1816

174. Pagrus caeruleostictus (Valenciennes, 1830)

175. Pagrus pagrus (Linnaeus, 1758)

SARPA Bonaparte, 183

176. Sarpa salpa (Linnaeus, 1758)

SPARUS Linnaeus, 1758

177. Sparus aurata Linnaeus, 1758

SPICARA Rafinesque, 1810

178. Spicara flexuosa Rafinesque, 1810

179. Spicara maena (Linnaeus, 1758)

180. Spicara smaris (Linnaeus, 1758)

SPONDYLIOSOMA Cantor, 1849

181. Spondyliosoma cantharus (Linnaeus, 1758)

SCIAENIDAE

ARGYROSOMUS De La Pylaie, 1835

182. Argyrosomus regius (Asso, 1801)

SCIAENA Linnaeus, 1758

183. Sciaena umbra Linnaeus, 1758

UMBRINA Cuvier, 1816

184. Umbrina cirrosa (Linnaeus, 1758)

MULLIDAE

MULLUS Linnaeus. 1758
185. Mullus barbatus Linnaeus, 1758

186. Mullus surmuletus Linnaeus, 1758

\section{CEPOLIDAE}

CEPOLA Linnaeus, 1766

187. Cepola macrophthalma (Linnaeus, 1758)

\section{POMACENTRIDAE}

CHROMIS Cuvier, 1814

188. Chromis chromis (Linnaeus, 1758)

\section{LABRIDAE}

CORIS Lacepède, 1801

189. Coris julis (Linnaeus, 1758)

LABRUS Linnaeus, 1758

190. Labrus merula Linnaeus, 1758

191. Labrus mixtus Linnaeus, 1758

192. Labrus viridis Linnaeus, 1758

SYMPHODUS Rafinesque, 1810

193. Symphodus cinereus (Bonnaterre, 1788)

194. Symphodus doderleini Jordan, 1891

195. Symphodus mediterraneus (Linnaeus, 1758)

196. Symphodus ocellatus (Forsskål, 1775)

197. Symphodus roissali (Risso, 1810)

198. Symphodus rostratus (Bloch, 1797)

199. Symphodus tinca (Linnaeus, 1758)

THALASSOMA Swainson, 1839

200. Thalassoma pavo (Linnaeus, 1758)

XYRICHTHYS Cuvier, 1814

201. Xyrichthys novacula (Linnaeus, 1758)

SCARIDAE

SPARISOMA Swainson, 1839

202. Sparisoma cretense (Linnaeus, 1758)*

\section{TRACHINIDAE}

ECHICHTHYS Bleeker, 1861

203. Echichthys vipera (Cuvier, 1829)

TRACHINUS Linnaeus, 1758

204. Trachinus araneus Cuvier, 1829

205. Trachinus draco Linnaeus, 1758

206. Trachinus radiatus Cuvier, 1829

TETRAGONURIDAE

TETRAGONURUS Risso, 1810

207. Tetragonurus cuvieri Risso, 1810

URANOSCOPIDAE 
URANOSCOPUS Linnaeus, 1758

208. Uranoscopus scaber Linnaeus, 1758

\section{BLENNIIDAE}

PARABLENNIUS Miranda-Ribeiro, 1915

209. Parablennius gattorugine (Linnaeus, 1758)

210. Parablennius sanguinolentus (Pallas, 1814)

211. Parablennius tentacularis (Brünnich, 1768)

SALARIA Forsskål, 1775

212. Salaria basilisca (Valenciennes, 1836)

213. Salaria pavo (Risso, 1810)

SCARTELLA Jordan, 1886

214. Scartella cristata (Linnaeus, 1758)

\section{CALLIONYMIDAE}

CALLIONYMUS Linnaeus, 1758

215. Callionymus lyra Linnaeus, 1758

216. Callionymus maculatus Rafinesque, 1810

217. Callionymus risso Lesueur, 1814

\section{GOBIIDAE}

GOBIUS Linnaeus, 1758

218. Gobius bucchichi Steindachner, 1870

219. Gobius niger Linnaeus, 1758

KNIPOWITSCHIA Iljin, 1927

220. Knipowitschia panizzae (Verga, 1841)

\section{SIGANIDAE}

221. Siganus rivulatus Forsskål, 1775*

222. Siganus luridus (Rüppell, 1829)*

LUVARIDAE

LUVARUS Rafinesque, 1810

223. Luvarus imperialis Rafinesque, 1810

\section{SPHYRAENIDAE}

SPHYRAENA Artedi, 1793

224. Sphyraena sphyraena (Linnaeus, 1758)

\section{GEMPYLIDAE}

RUVETTUS Cocco, 1833

225. Ruvettus pretiosus Cocco, 1833*5

TRICHIURIDAE

LEPIDOPUS Goüan, 1770

226. Lepidopus caudatus (Euphrasen, 1788)

TRICHIURUS Linnaeus, 1758

227. Trichiurus lepturus Linnaeus, 1758

\section{XIPHIIDAE}

XIPHIAS Linnaeus, 1758

228. Xiphias gladius Linnaeus, 1758

\section{SCOMBRIDAE}

AUXIS Cuvier, 1829

229. Auxis rochei (Risso, 1810)

EUTHYNNUS Lütken, 1883

230. Euthynnus alletteratus (Rafinesque, 1810)

SARDA Cuvier, 1829

231. Sarda sarda (Bloch, 1793)

SCOMBER Linnaeus, 1758

232. Scomber colias Gmelin, 1789

233. Scomber scombrus Linnaeus, 1758

THUNNUS South, 1845

234. Thunnus thynnus (Linnaeus, 1758)

CENTROLOPHIDAE

CENTROLOPHUS Lacepède, 1802

235. Centrolophus niger (Gmelin, 1789)

\section{STROMATEIDAE}

STROMATEUS Linnaeus, 1758

236. Stromateus fiatiola Linnaeus, 1758

\section{ORDER: PLEURONECTIFORMES}

CITHARIDAE

CITHARUS Rose, 1793

237. Citharus linguatula (Linnaeus, 1758)

\section{SCOPHTHALMIDAE}

LEPIDORHOMBUS Günther, 1862

238. Lepidorhombus boscii (Risso, 1810)

239. Lepidorhombus whiffiagonis (Walbaum, 1792)

ZEUGOPTERUS Gottsche, 1835

240. Zeugopterus regius (Bonnaterre, 1788)

SCOPHTHALMUS Rafinesque, 1810

241. Scophthalmus maximus (Linnaeus, 1758)

242. Scophthalmus rhombus (Linnaeus, 1758)

\section{BOTHIDAE}

ARNOGLOSSUS Bleeker, 1862

243. Arnoglossus imperialis (Rafinesque, 1810)

244. Arnoglossus kessleri Schmidt, 1915

245. Arnoglossus laterna (Walbaum, 1792)

246. Arnoglossus rueppelli (Cocco, 1844)

247. Arnoglossus thori Kyle, 1913 
BOTHUS Rafinesque, 1810

248. Bothus podas (Delaroche, 1809)

\section{PLEURONECTIDAE}

PLATICHTHYS Girard, 1856

249. Platichthys flesus (Pallas, 1811)

SOLEIDAE

BUGLOSSIDIUM Chabanaud, 1930

250. Buglossidium luteum (Risso, 1810)

MICROCHIRUS Bonaparte, 1833

251. Microchirus ocellatus (Linnaeus, 1758)

252. Microchirus variegatus (Donovan, 1808)

MONOCHIRUS Rafinesque, 1814

253. Monochirus hispidus Rafinesque, 1814

PEGUSA Günther, 1862

254. Pegusa lascaris (Risso, 1810)

255. Pegusa nasuta (Pallas, 1814)

256. Pegusa impar Bennett, 1831

SOLEA Quensel, 1806

257. Solea solea (Linnaues, 1758)
SYNAPTURICHTHYS Chabanaud, 1927

258. Synapturichthys kleini (Risso, 1827)

ORDER: TETRAODONTIFORMES

BALISTIDAE

BALISTES Linnaues, 1758

259. Balistes carolinensis Gmelin, 1789

\section{TETRAODONTIDAE}

LAGOCEPHALUS Swainson, 1839

260. Lagocephalus sceleratus (Gmelin, 1789)*6

MOLIDAE

MOLA Koelreuter, 1770

261. Mola mola (Linnaeus, 1758)

RANZANIA Nardo, 1840

262. Ranzania laevis (Pennant, 1776)

* New species records - personal observations except ${ }^{1}$ BAKIU et al. (2018); ${ }^{2}$ GIOVOS \& CAKALLI (2017); ${ }^{3}$ BAKIU (2019); ${ }^{4}$ BAKIU \& TROPLINI (2018); ${ }^{5}$ GIOVOS \& BAKIU (2017); ${ }^{6}$ BAKIU \& DURMISHAJ (2019).

\section{REFERENCES}

BAKIU, R. 2019. First record of the milk shark Rhizoprionodon acutus (Rüppell, 1837) from Albanian waters. In Collective Article: New Mediterranean Biodiversity Records (April 2019). Mediterranean Marine Science, 20 (1): 238.

BAKIU, R. \& E. TROPLINI, 2018. First record of the great torpedo ray Tetronarce nobiliana (Bonaparte, 1835) in the Albanian waters. In Collective Article: New Mediterranean Biodiversity Records (November 2018), Mediterranean Marine Sciences, 19(3): 680.

BAKIU, R \& S. DURMISHAJ, 2019. First record of the silver-cheeked toadfish Lagocephalus sceleratus (Gmelin, 1789) in Albanian waters. In Collective Article: New Mediterranean Biodiversity Records (April, 2019), Mediterranean Marine Sciences, 20(1): 237238.

BAKIU, R. \& I. CAKALLI \& I. GIOVOS, 2018. The first record of bigeyed sixgill shark, Hexanchus nakamurai Teng, 1962 in Albanian waters. Journal of the Black Sea Mediterranean Environment, 24 (1): 74-79.
FRICKE, R., ESCHMEYER, W. N. \& R. VAN DER LAAN, (Editors) 2020. Eschmeyer's Catalog of Fishes: Genera, Species, References. Electronic version accessed 10/09/2020.

GIOVOS, I. \& R. BAKIU. 2017. First record of the Oilfish, Ruvettus pretiosus (Cocco, 1833) in Albanian waters. In Collective Article: New Mediterranean Biodiversity Records (July, 2017), Mediterranean Marine Sciences, 18(2): 364-365.

GIOVOS, I. \& M. CAKALLI 2017. First record of the Bigeye Thresher Alopias superciliosus in Albanian waters. In Collective Article: New Mediterranean Biodiversity Records. Mediterranean Marine Sciences, 18(3): 541-542.

KOVAČIĆ, M., L. LIPEJ \& J. DULČIĆ. 2020. Evidence approach to checklists: critical revision of the checklist of the Adriatic Sea fishes. Zootaxa, 4767 (1): 1-55.

IHO (International Hydrographic Organization). 1953. Limits of Oceans and Seas, 3rd edition. Special Publication No. 23 (S-23). Monaco: International Hydrographic Organization. 
LIPEJ, L. \& J. DULČIĆ. 2010. Checklist of the Adriatic Sea Fishes. Zootaxa, 2589 (1): 1-92

RAKAJ, N. 1995. Iktiofauna e Shqiperise (Ichthyofauna of Albania). Sheptia Botuese Libri Universitar: 700 pp (in Albanian)

SIMEONI, U., N. PANO \& P. CIAVOLA. 1997. The coastline of Albania: morphology, evolution and coastal management issues. CIESM Sci- ence Series No. 3, Transformation and evolution of the Mediterranean coastline. Bulletin de 1'Institut Ocranographique, Monaco, No. Special 18, 1987: 151-168.

VAN DER LAAN, R., R. FRICKE \& W. N. ESCHMEYER, (Editors) 2020. Eschmeyer's Catalog of Fishes: Classification. Electronic version accessed 10/09/ 2020.

Received:11 September 2020

Accepted: 29 May 2021 


\title{
Pregledna lista morskih riba Albanije
}

\author{
Alen SOLDO i Rigers BAKIU
}

\section{SAŽETAK}

U ovom se radu prezentira nova pregledna lista morskih riba koje žive u morima Albanije, a ujedno je i prva takva lista publicirana na engleskom jeziku. Lista sadrži 262 vrste riba i sastavljena je korištenjem objavljene literature te podacima iz istraživanja autora.

Ključne riječi: lista; ribe; Albanija; Jadransko i Jonsko more 